\title{
Trace mercury determination in drinking and natural water after preconcentration and separation by DLLME-SFO method coupled with cold vapor atomic absorption spectrometry
}

\author{
Atousa Abdollahi ${ }^{1}$, Mooud Amirkavehei ${ }^{2}$, Mohammad Mehdi Gheisari ${ }^{3}$ and Fariba Tadayon ${ }^{4}$ \\ ${ }^{1}$ Department of Environmental Chemistry, Islamic Azad University Khorasgan (Isfahan) Branch, Iran \\ ${ }^{2}$ Department of Environmental Chemistry, Islamic Azad University Khorasgan (Isfahan) Branch, Iran \\ ${ }^{3}$ Department of Environmental Chemistry, Islamic Azad University Khorasgan (Isfahan) Branch, Iran \\ ${ }^{4}$ Department of Chemistry, Islamic Azad University North Tehran Branch,Tehran, Iran
}

\begin{abstract}
A novel dispersive liquid-liquid microextraction based on solidification of floating organic drop (DLLME-SFO) for simultaneous separation/preconcentration of ultra trace amounts of mercury was used. A method based on amalgamation was used for collection of gaseous mercury on gold coated sand (Gold trap). The concentration of mercury was determined by cold vapor atomic absorption spectrometry (CV-AAS). The DLLME-SFO behavior of mercury by using dithizone as complexing agent was systematically investigated. The factors influencing, the complex formation and extraction of DLLME-SFO method such as type and volume of extraction and disperser solvents, $\mathrm{pH}$, concentration of salt, centrifuging time and concentration of the chelating agent were optimized. The method was successfully applied to the determination of mercury in drinking and natural water and satisfactory relative recoveries (95-105\%) were achieved. The proposed procedure was based on very low consumption of organic solvents. The other benefits of the system were sensitive, simple, friendly to the environment, rejection of matrix constituent, low cost, the time consuming and high enrichment factor.
\end{abstract}

Keywords: mercury; Dispersive liquid-liquid microextraction based on solidification of floating organic drop (DLLME-SFO); Dithizone; Cold vapor atomic absorption spectrometry (CV-AAS)

\section{Introduction}

Mercury $(\mathrm{Hg})$ is known as a harmful metal and its toxic effect has been known for centuries. A considerable amount of mercury comes from anthropogenic sources such as manufacture of plastic, paper, paint, pharmaceutical (Craig, 1986). Organometalic species of mercury are more toxic than inorganic, because of their high affinity to $\mathrm{SH}$ group of proteins and lipid issues (Pongratz et al, 1999). One of the routes of intake of this metal in to the human body is drinking water (Clifton, 2007); however, as the concentration of $\mathrm{Hg}$ in environmental and biological samples is very low, its direct determination is difficult even with highly sensitive and selective analytical techniques. Thus, a separation and pre- concentration steps prior to its determination is required. The widely used techniques for the separation and preconcentration of $\mathrm{Hg}$ are traditional liquid-liquid extraction (Gaona et al, 2003), solid phase microextraction (SPE) (Hashempur et al, 2008), single drop microextraction (SDME) (Pena-Pereira et al, 2009), dispersive liquid- liquid microextraction (DLLME) (Gaoet et al, 2011)] and solidified of floating organic drop microextraction (López-García et al, 2010) have been recently developed.

A novel dispersive liquid-liquid micro extraction method based on the solidification of floating organic drop (DLLME-SFO) was introduced by Leong et al (2008). It is based on DLLME and the solidification of floating organic drop. In the DLLME-SFO procedure, selecting a perfect extraction solvent is vital. It should have low solubility in water, high affinity to analytes, low melting point below room temperature, and lower density than water. The large contact surface between the sample and the droplets of extractants speeds up mass transfer, as fast as DLLME and shorter extraction time than LLME-SFO. In this method there is no need to use conical bottom glass tubes, which are easily damaged and 
hard to clean. The floated extractant is solidified and is easily collected for analysis. The advantages of DLLME-SFO method are simplicity of operation, rapidity, low cost, high recovery, and using extracting solvent with lower density rather than water and lower toxicity in contrast with DLLME. In this study, dithizone, the classical organic reagent was selected as chelating reagent and a new DLLME-SFO method combined cold vapor atomic absorption spectroscopy (CV-AAS) was developed for separation, enrichment and determination of mercury in water samples and fish. Factors affecting the extraction efficiency, such as solution $\mathrm{pH}$, concentration of organic ligand, extraction time, sample volume, nature of organic solvent and its volume, and kind of disperser solvent and its volume were studied and optimized.

\section{Materials and Methods}

All reagents were used of analytical reagent grades and were purchased from the Merck Company (Darmstadt, Germany). The $\mathrm{pH}$ of sample that containing proper amount of $\mathrm{Hg}$ was adjusted to $\sim 7$ by using $\mathrm{HCl}$ solution and $\mathrm{NaOH}$ solution. About $10 \mathrm{ml}$ of the solution was transferred in to $\sim 12 \mathrm{ml}$ test tube and dithizone solution as complexing agent was added and mixtured, then extraction solvent and dispersive solvent was rapidly injected in to the aqueous sample. In this stage a cloudy solution was formed in a test tube the ions reacted with dithizone and were extracted in to extraction solvent in a few seconds. After centrifuging, the organic solution droplet was floated on the surface of the aqueous solution due to low density below water. The test tube was there after put into an ice bath for $5 \mathrm{~min}$. At this time the floated solvent was solidified because of the low melting point $\left(24^{\circ} \mathrm{C}\right)$, then the solidified solvent was transferred in to a conical vial where it melted immediately, it was manually injected to gold trap cold vapor atomic absorption spectrometer system for the determination of mercury.

\section{Results and Discussion}

In order to obtain a high enrichment factor, different parameters affecting the complex formation, extraction and analytes determination were optimized by using the uni variable approach. In DLLME-SFO procedure, selecting a perfect extraction solvent is vital. Accordingly, several extraction solvent, including 1-undecanol, 1-dodecanol, 1,10dichlrodecan and n-hexadecan were investigated. Suitable extraction solvent was recognized. The effect of the extraction volume on the extraction was also investigated.

Selection of the disperser solvent considers its miscibility with extraction solvent and water. In this experminet acetone, ethanol, methanol and acetonitrile were tested. With acetonitrile emulsion formed and separation of two phase was difficult and proper solvent was selected. The effect of the volume of disper solvent in the range of $50-400 \mu \mathrm{L}$ on the extraction efficiency of analytes was examined. The results show that by increasing of the disperser solvent until $200 \mu \mathrm{L}$ the absorbance of analyte was maximized. At the low volume of disperser solvent, extraction solvent wasn't completely dispersed and the extract efficiency wasn't complet. In the volume of disperser solvent greater than $300 \mu \mathrm{L}$, the extraction efficiency was decreased because of the increase in solution of complex of analytes in aqueous phase containing high percentage of disperser solvent. (Fig. 1)

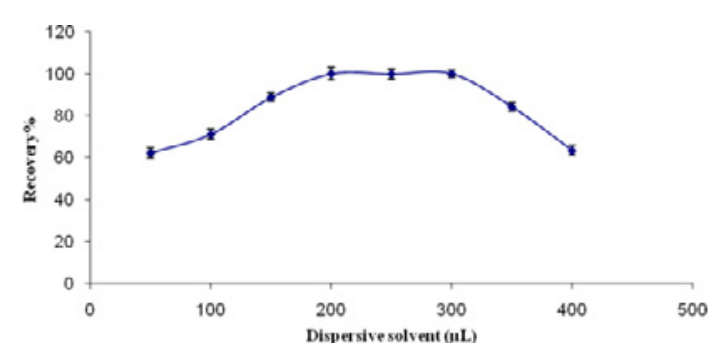

Fig. 1. Effect of disperser solvent volume.

The $\mathrm{pH}$ of the sample solution is one of the important factors affecting the formation of complex and subsequent extraction in DLLME -SFO. The effect of $\mathrm{pH}$ on the extraction of $\mathrm{Hg}$ with dithizone as a complex agent was studied by varying the $\mathrm{pH}$ within the range of 3-11.The $\mathrm{pH}$ was adjusted by using either $\mathrm{HCl}$ solution of $0.05 \mathrm{~mol} \mathrm{l}^{-1}$ or $\mathrm{NaOH}$ solution $0.05 \mathrm{~mol} \mathrm{l}^{-1}$, while the other variable keeping constant. The results illustrated in Fig (2) demonstrating that the analytical signal is nearly constant in the $\mathrm{pH}$ range of $\mathrm{pH} 3-11$.

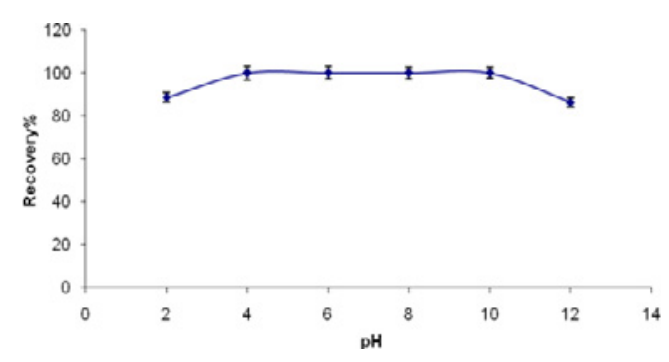

Fig. 2. Effect of $\mathrm{pH}$.

An important parameter which influences the increase in the ratio of volume of aqueous phase to organic phase will result in significant increase in the enrichment factor, but an increase in sample volume may cause a decrease in extraction efficiency in a given time. The effect of sample volume on extraction of $\mathrm{Hg}$ from different sample volume $(5-50 \mathrm{ml})$, in a proper size vial, was investigated. The results Fig (3) showed that the extraction was quantitative with the aqueous phase volume in the range of $5-40 \mathrm{ml}$ and a decrease was observed with further increase in sample volume. Because of good sensitivity of CV-AAS for simple work, the volume of $10 \mathrm{ml}$ was used. 


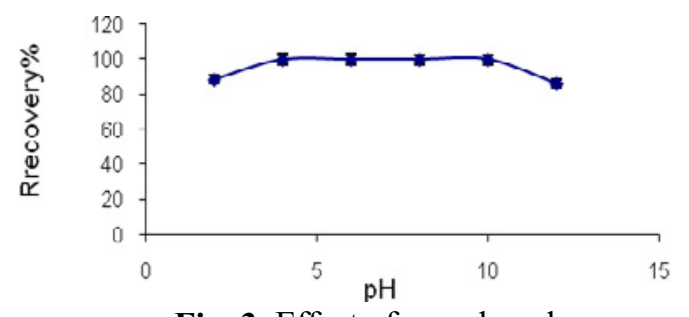

Fig. 3. Effect of sample volume

The effect of DZ concentration on the extraction efficiency was evaluated. Optimum concentration was selected for further studies.

In DLLME-SFO, extraction time is defined as the interval time between the injection of the solution of disperser and extraction solvent before starting to centrifuge. The effect of the extraction time was examined in the range of $0-5 \mathrm{~min}$ with constant experimental conditions. As a result extraction time has no impact on extraction efficiency. In this method after the formation of a cloudy solution, the surface area between the extraction solvent and aqueous phase is very large. Thereby transition of the complex from aqueous phase to the extraction solvent is fast. Thus, the most important advantage of DLLME-SFO is time independence of the method. In this method time consuming steps are centrifuging of the sample solution in the extraction procedure, which is about $3 \mathrm{~min}$ and solidified of 1 -undecanol, which is about $5 \mathrm{~min}$.

Some metal ions also form stable chelates with dithizone, being a potential source of interference with the analytical procedure for mercury species. So the effect of common coexisting ions $\left(\mathrm{Cu}^{+2}, \mathrm{Zn}^{+2}, \mathrm{~Pb}^{+2}, \mathrm{Cd}^{+2}, \mathrm{Al}^{+3}\right)$ on mercury species analysis was investigated.

To examine the reliability of the recommended procedure, the method was applied for the deteremination of mercury in tap water, well water, spring water, sea water, river water and fish.

\section{Conclusion}

It has been demonstrated that dispersive liquid liquid liquid microextraction- solidified floating organic drop combined with gold trap CV-AAS can be used for separation/ enrichment and determination of ultra trace of mercury. Further DZ as chelating agent can be formation complex with mercury and extracted it to extraction solvent. Thus this method permits effective separation and concentration of $\mathrm{Hg}$ and final determination by $\mathrm{CV}$-AAS and provide a novel route for trace determination mercury in several categories of natural waters. The most important advantages of DLLME-SFO are; lower time of detect, very short extraction time and ease of operation. It is inexpensive and has higher enrichment factor.

\section{References}

Craig P.J. Organometallic Compounds in the Environment, Longman, London, 1986.

Clifton JC, 2nd. Mercury exposure and public health. Pediatr Clin North Am 2007;54: 237-69.

Gaona X, Valiente M. Stability study on a Westöö-based methodology to determine organomercury compounds in polluted soil samples. Anal. Chim. Acta 2003; 480: 219-230.

Gao Zh, Ma Xi. Speciation analysis of mercury in water samples using dispersive liquid-liquid microextraction combined with high-performance liquid chromatography. Anal. Chim. Acta 2011; 702:50-55.

Hashempur T, Rofouei M.K, Khorrami, A.R. Speciation analysis of mercury contaminants in water samples by RP-HPLC after solid phase extraction on modified $\mathrm{C} 18$ extraction disks with 1,3-bis(2-cyanobenzene)triazene. Microchem. J 2008; 89: 131-136.

López-García I, Rivas R. E, „Hernández-Córdoba M. Liquid-phase microextraction with solidification of the organic floating drop for the preconcentration and determination of mercury traces by electrothermal atomic absorption spectrometry. Anal Bioanal Chem 2010; 396:3097-3102.

Leong M.I, Huang S.D. Dispersive liquid-liquid microextraction method based on solidification of floating organic drop combined with gas chromatography with electron-capture or mass spectrometry detection . J. Chromatogr. A 2008; 1211: 8 .

Pongratz R. Heumann K.G. Production of methylated mercury, lead, and cadmium by marine bacteria as a significant natural source for atmospheric heavy metals in polar regions. Chemosphere 1999; 39: 89-102.

Pena-Pereira F, Bendicho I. C, Vidal L. A. Canals, Speciation of mercury by ionic liquid-based single-drop microextraction combined with high-performance liquid chromatography -photodiode array detection. Talanta 2009; 78: 537-541. 